\title{
Aportes de la conectividad cerebral a la comprensión y el diagnóstico de la enfermedad mental
}

\section{Contributions of brain connectivity to the understanding and diagnosis of mental illness}

\author{
Cecilia María Díaz-Soto, ${ }^{\star}$ Paulina Vélez-Gómez ${ }^{\ddagger}$ \\ * Escuela de Graduados, Doctorado en Ciencias de la Salud. \\ ‡ Facultad de Psicología.
}

Universidad CES. Medellín, Colombia.

\section{RESUMEN}

Los trastornos mentales graves continúan desafiando y preocupando a los profesionales e investigadores de la salud mental. La búsqueda sobre la etiología básica, la fisiopatología fundamental y el tratamiento definitivo para éstos aún continúa. Las tecnologías de neuroimagen contribuyen a la comprensión de los trastornos mentales y pueden emerger como una herramienta de diagnóstico. Para construir el artículo, se rastreó información sobre cómo se ha estudiado la enfermedad mental grave por medio de la conectividad cerebral en la base de datos Web of Science; luego de obtener los resultados, se ingresaron a la plataforma Tree of Science para su análisis bibliográfico. Se trazó una óptica general del tema conectividad cerebral en la enfermedad mental y se exponen algunos de los hallazgos más importantes en esta búsqueda. Se discuten algunos aspectos importantes, como el hecho de que los mecanismos biológicos que se han propuesto para entender los trastornos mentales, principalmente del estado de ánimo, por lo general no habían incluido un componente neuropatológico. Finalmente, se concluye que es necesario el abordaje de estos trastornos desde perspectivas transdisciplinares como la neuropsiquiatría.

Palabras clave: Conectividad, psicopatología, esquizofrenia, trastorno bipolar, depresión, resonancia magnética funcional, revisión.

\section{ABSTRACT}

Severe mental disorders continue to challenge and worry mental health professionals and researchers. The search for the underlying etiology, the fundamental pathophysiology, and the definitive treatment for these is still ongoing. Neuroimaging technologies contribute to the understanding of mental disorders and may emerge as a diagnostic tool. For this article, information on how severe mental illness has been studied using brain connectivity was tracked in the Web of Science database. After obtaining the results, the Tree of Science platform was used for bibliographic analysis. A general view of the topic of brain connectivity in mental illness was drawn, and some of the most critical findings in this search are presented. Some important aspects are discussed, such as the fact that the biological mechanisms that have been proposed to understand mental disorders, mainly mood disorders, generally had not included a neuropathological component. Finally, it is concluded that it is necessary to approach these disorders from transdisciplinary perspectives such as neuropsychiatry.

Keywords: Connectivity, psychopathology, schizophrenia, bipolar disorder, depression, functional magnetic resonance imaging, review.

\section{INTRODUCCIÓN}

Las condiciones que llamamos enfermedad mental, aquellos trastornos mentales que interrumpen y desestabilizan la vida durante años, continúan desafiando y preocupando a las profesiones relacionadas con la salud mental. La búsque- da sobre la etiología básica, la fisiopatología fundamental y el tratamiento definitivo para trastornos como la esquizofrenia, el trastorno bipolar y la depresión aún continúan.

No obstante, se han producido profundos cambios en el conocimiento sobre trastornos mentales graves, los esquemas actuales de clasificación diagnóstica com- 
prenden una colección de criterios diagnósticos teóricos, prototípicos, categóricos, operacionalizados, que tienen utilidad clínica y han conducido a hipótesis neurobiológicas interesantes. Después de revisar el desarrollo y el estado actual del diagnóstico de trastornos mentales, parece que los esfuerzos empíricos para dilucidar otros componentes conductuales y construcciones dimensionales más importantes de los trastornos mentales, se han hecho gracias a la investigación neurobiológica. ${ }^{1}$

Con respecto a estas investigaciones, está claro que las tecnologías de neuroimagen contribuyen a la comprensión de los trastornos mentales y pueden emerger como una herramienta de diagnóstico, incluso se han propuesto biomarcadores de imágenes para la evaluación de enfermedades y para guiar los procedimientos terapéuticos. ${ }^{1}$ Uno de los objetivos principales de la investigación en neurociencia humana es establecer las relaciones entre las estructuras cerebrales y sus funciones, ${ }^{2}$ una de las formas utilizadas para este fin es la conectividad funcional, que ha sido definida como la correlación temporal de un índice neurofisiológico medido en diferentes áreas del cerebro. ${ }^{3,4}$

Por consiguiente, el objetivo de este artículo es ayudar en la construcción de un marco de referencia acerca de las contribuciones que han hecho la neurociencia y las técnicas de conectividad cerebral para la comprensión de la enfermedad mental.

\section{METODOLOGÍA}

Para construir el artículo se rastreó información sobre cómo se ha estudiado la enfermedad mental grave por medio de la conectividad cerebral. Inicialmente se utilizó la base de datos Web of Science, con el fin de identificar los artículos que contenían información sobre la conectividad cerebral y la enfermedad mental grave, para lo que se utilizó la ecuación de búsqueda (EB): Tema = (functional connectivity) AND Tema $=$ (Mental illness) con rangos de fecha entre enero de 2001 y enero de 2019.

Luego de obtener los resultados se ingresaron a la plataforma Tree of Science (ToS) (árbol de la ciencia); ${ }^{5}$ esta herramienta permite hacer la construcción de un estado del arte por medio de un algoritmo basado en teoría de grafos, el cual identifica a los artículos como nodos, éstos representan unidades de conocimiento que se enlazan entre ellos por medio de las citaciones, las que representarán los links entre los nodos, de los cuales se identificarán los más importantes a partir de las conexiones que tengan con otros nodos. ${ }^{6}$ Esta técnica permite hacer una visualización de la información científica y la gráfica en forma de árbol, ubicando así en las raíces las referencias que fundamentan la conectividad cerebral en enfermedad mental; en el tronco se ubican las referencias consideradas estructurales y, finalmente, en las hojas se ubicará el panorama actual con respecto al tema (Figura 1).

\section{RESULTADOS}

\section{Raíces}

A partir de la identificación de los artículos, se registraron aspectos importantes de la conectividad cerebral en la enfermedad mental, con el fin de trazar una óptica general del tema haciendo una revisión de algunos elementos fundamentales con relación a éste.

Es así como en las raíces se encontró un artículo que exponía las ventajas potenciales de un enfoque basado

\section{Figura 1:}

ToS de la ecuación de búsqueda. Fuente: Elaboración propia a partir de los resultados de la ecuación de búsqueda en Web of Science y la utilización de Tree of Science.
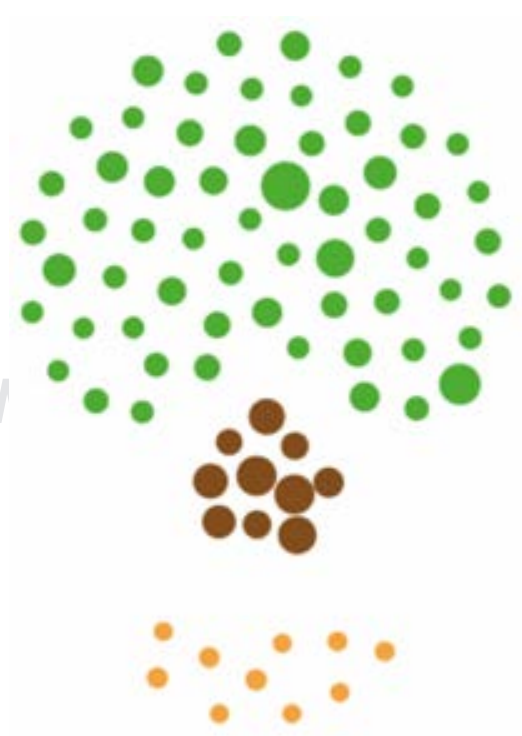

$\Gamma$

Yu Q, Du Y, Chen J, Sui J. Application of graph theory to assess static and dynamic brain connectivity: approaches for building brain graphs. Proc IEEE Inst Electr Electron Eng. 2018; 106 (5): 886-906.

L - - - - - - - -

$\Gamma_{\text {Buckholtz JW, Meyer-Lindenberg A. Psychopatholo- }}$ gy and the human connectome: toward a transdiagnostic model of risk for mental illness. Neuron. 2012; 74 (6): 990-1004. doi: 10.1016/j.neuron.2012.06.002.

L

Raichle ME, MacLeod AM, Snyder AZ, Powers WJ, Gusnard DA, Shulman GL. A default mode of brain function. Proc Natl Acad Sci U S A. 2001; 98 (2): 676-682. 
en la neurociencia para la clasificación de la enfermedad mental, en el que muestra que no existe consenso sobre cómo lograr este objetivo, teniendo como base que existen cinco criterios para validar los diagnósticos clínicos en psiquiatría que son la descripción clínica, pruebas de laboratorio, delimitación, estudios de seguimiento y datos familiares, donde el objetivo es especificar el pronóstico, preguntándose, además, cuándo y cómo construir un marco a largo plazo para la investigación que podría generar descubrimientos basados en clasificación en genómica y neurociencia, así como observación clínica, con el objetivo de mejorar los resultados del tratamiento. ${ }^{7}$

Por otro lado, Friston y Frith, en 1995, revisaron la evidencia de cambios fisiopatológicos de los lóbulos prefrontal y temporal en los sujetos esquizofrénicos y de integración anormal de la dinámica fisiológica en estas dos regiones, con el argumento de que algunos fenómenos esquizofrénicos se entienden mejor en términos de interacciones anormales entre diferentes áreas cerebrales, y no sólo los niveles de fisiología y anatomía funcional. ${ }^{4}$ Este trabajo concluyó que la actividad cerebral generada intrínsecamente y la integración de ésta en el dominio perceptivo depende de las interacciones coherentes entre las cortezas prefrontales y las dedicadas a las representaciones perceptivas. Así mismo, muchos signos y síntomas que experimentan los pacientes con diagnóstico de esquizofrenia influyen en su relación con los demás, ya que ésta está mediada por el lenguaje y la expresión, o su ausencia. Un ejemplo de esto es la pobreza del habla, un afecto inadecuado o plano, habla incoherente, disminución del contenido del habla y las alucinaciones auditivas, esto debido a que la anatomía funcional de las representaciones perceptivas relacionadas con el lenguaje se centra en las regiones temporales.

Finalmente, una revisión que se realizó en el Departamento de Psicología de la Universidad de Harvard definió que luego de varios años de investigación en imágenes cerebrales, se logró definir y proporcionar evidencia de que la red predeterminada es relevante para la comprensión de trastornos mentales como el autismo, la esquizofrenia y la enfermedad de Alzheimer. Esta red es un sistema cerebral específico, anatómicamente definido y preferentemente activo cuando las personas no están enfocadas en el entorno externo, cuya función es estar activa cuando los individuos se dedican a tareas enfocadas internamente, incluida la recuperación de memoria autobiográfica, previendo el futuro y concibiendo las perspectivas de otros. ${ }^{8}$

Por otra parte, Buckner y colaboradores afirmaron que el subsistema del lóbulo temporal medial proporciona información de experiencias anteriores en forma de memorias y asociaciones, componentes básicos de la simulación mental y que el subsistema prefrontal medial facilita el uso flexible de esta información durante la construcción de simulaciones mentales relevantes; ${ }^{8}$ la convergencia de ambos en nodos importantes de integración que incluyen la corteza cingulada posterior, tiene implicaciones funcionales y anatómicas con respecto a los roles de adaptación de la red predeterminada para usar las experiencias pasadas para planificar el futuro, navegar por las interacciones sociales y maximizar la utilidad de los momentos en que el mundo externo no nos involucra.

\section{Tronco}

Los artículos ubicados en el tronco son aquellos que estructuran la evidencia de que los estudios de conectividad cerebral podrían objetivar las áreas cerebrales que presentan disfunción en las diferentes enfermedades mentales y sus manifestaciones, encontrando una posible etiología neurobiológica para éstas.

Así, por ejemplo, se encontró un estudio cuyo objetivo fue revisar la evidencia disponible para la patología de la sustancia blanca en la esquizofrenia, el cual examina su posible papel como sustrato de la conectividad dañada. Los datos neuropatológicos hallados sugieren anomalías en la estructura y función glial, como también en estructuras mielinizadas. Algunos de estos hallazgos asocian esta enfermedad con una interrupción significativa en la expresión de genes de mielinización, como también cambios en la microestructura de la materia blanca en la esquizofrenia, mencionando que las estructuras mielinizadas son un candidato para un sustrato anatómico para la desconexión, y pueden actuar de forma sinérgica con los cambios sinápticos para producir una desconexión funcional. Aunque la relación entre los cambios de la sustancia blanca y gris en la enfermedad sigue sin estar clara, la evidencia emergente apunta a que los sustratos neuronales de la conectividad tienen un papel clave en el desarrollo de la enfermedad. ${ }^{9}$

Por otro parte, Buckholtz y Meyer-Lindenberg propusieron un modelo de transdiagnóstico de riesgo para enfermedades mentales, argumentando que las diversas formas de psicopatología se caracterizan por fallas en la interacción de regiones específicas del cerebro; estos hallazgos se han dado gracias a las imágenes cerebrales funcionales que han proporcionado información sobre la naturaleza de dicha desconectividad del cerebro. Finalmente, proponen factores de riesgo genéticos y ambientales que inciden en los circuitos a nivel de sistemas para varias dimensiones centrales de la cognición, produciendo síntomas transdiagnósticos. ${ }^{10}$

Por esta misma época, en el Departamento de Psiquiatría de la Universidad de Yale, realizaron una revisión donde se expone el papel de la desactivación de la red predeterminada (DMN) en la cognición y la enfermedad. A través de técnicas de neuroimagen como la rs-fcMRI, se encontró implicada la red predeterminada en alteraciones cognitivas y síntomas asociados con trastornos 
neuropsiquiátricos como la esquizofrenia y la depresión, dos condiciones que muestran una falla potencial para suprimir las señales de DMN. Por su parte, los individuos con diagnóstico de trastorno depresivo no logran reducir la actividad de $\mathrm{DMN}$ al reevaluar las imágenes negativas, estas deficiencias de supresión de DMN en la depresión se han relacionado con la rumiación negativa, a diferencia de la esquizofrenia, donde la falta de supresión de DMN se ha identificado durante tareas cognitivamente exigentes. ${ }^{11}$

Así mismo, Anticevic y colaboradores, en un estudio que intentaba caracterizar las alteraciones talamocorticales en la esquizofrenia y la enfermedad bipolar, lograron confirmar cuantitativamente que la hiper/hipo conectividad talámica que encontraron el grupo de pacientes con esquizofrenia sigue una división anatómica general entre las redes sensoriales-motoras y las redes prefrontalestriatal-cerebelosa, encontrando patrones similares a éstos, pero moderados, en los pacientes con trastorno afectivo-bipolar. ${ }^{12}$

También, encontraron una correlación positiva y significativa entre la puntuación total de la escala PANSS (escala de síntomas positivos y negativos en esquizofrenia) y las regiones que muestran una conectividad excesiva, indicando que los pacientes con síntomas más severos exhiben una conexión talámica más fuerte con las cortezas sensoriales. Conforme a esto, la subescala de psicopatología general también mostró relaciones significativas con la sobreconectividad. Estos resultados destacan una relación entre los síntomas y la sobreconectividad talámica de todo el cerebro en la esquizofrenia, pero también sugieren que la gravedad de la enfermedad puede estar relacionada con la alteración talámica observada. Los autores concluyen que estos hallazgos constituyen alteraciones de la desconexión en las redes sensoriales-motoras frente a los ganglios prefrontal-estriatal-cerebeloso. ${ }^{12}$

\section{Ramas}

Finalmente, los artículos que su ubicaron en las ramas dan cuenta de las diferentes perspectivas desde donde ha sido abordado el tema de la enfermedad mental con la ayuda de técnicas de conectividad cerebral, que en su gran mayoría se trata de artículos de revisión, encontrando pocos artículos originales que hayan detallado estas técnicas utilizadas en investigaciones empíricas (Tablas 1 y 2).

Con respecto a los artículos originales donde se estudió la conectividad cerebral en pacientes con esquizofrenia, se encontró que, en su totalidad, se realizaron por medio de imágenes de resonancia magnética, tanto en presencia de una tarea cognitiva (dos artículos, 40\%), como en reposo. Estos estudios también se podrían dividir en términos generales si se estaban estudiando y encontrando anormalidades cerebrales regionales o si se estudiaron los circuitos cere- brales o la conectividad entre regiones cerebrales. En estos estudios se revelan resultados que coinciden en describir una conectividad alterada entre regiones del cerebro y la sintomatología asociada al trastorno, lo que apoyaría la hipótesis de otros investigadores, quienes afirman que los trastornos psicóticos se deben a una conectividad axonal anormal entre regiones corticales, lo que se ha generalizado en el concepto de desconectividad cerebral, común en la esquizofrenia. ${ }^{13}$

En el caso de los artículos donde se exponen resultados de estudios de conectividad cerebral en esquizofrenia y en otros trastornos mentales graves, se encontró que cuatro artículos (80\%) describen que la fMRI se realizó en estado de reposo. Los trastornos que se estudiaron fueron: un artículo (20\%) estudió el síndrome de fatiga crónica, dos artículos (40\%) se trataban de estudios en población con esquizofrenia, uno de ellos interesado en comparar esta población con sujetos con TAB y el otro buscaba evaluar pacientes con diagnóstico de esquizofrenia con antecedentes de maltrato infantil. Por otro lado, un artículo (20\%) estudió la afectividad negativa en el primer episodio depresivo de adolescentes y un artículo (20\%) mostró resultados de estudios realizados en población con exposición temprana a trauma.

Finalmente, uno de los hallazgos más interesantes de esta búsqueda bibliográfica fue el estudio de tratamiento realizado por un grupo de investigadores, en el que se exploró el valor predictivo de la actividad metabólica de la corteza cingulada anterior subgenual (sgACC) basal para el resultado clínico del tratamiento de los trastornos depresivos mayores y, posteriormente, probaron que una actividad metabólica sgACC basal más alta puede indicar un resultado clínico beneficioso para este tipo de tratamiento, proponiendo al sgACC como un marcador neurobiológico específico para la respuesta antidepresiva. ${ }^{14}$

A continuación, se revisaron los artículos que se trataban de revisiones de la literatura, tanto sistemáticas como narrativas, encontrando revisiones donde se describen anormalidades regionales del cerebro en otros trastornos mentales graves, como la depresión mayor.

Así, por ejemplo, en la revisión realizada por Pandya y colaboradores, se describen estudios donde se puntualizan anormalidades a nivel cortical, límbico subcortical, ganglios basales y tallo cerebral. Sin embargo, en esta revisión se describen hallazgos dispares del cambio en la actividad local en las regiones cerebrales en los diferentes estudios y grupos de sujetos, llevado a algunos investigadores a proponer que la anormalidad en la depresión y otros trastornos psiquiátricos pueden deberse a desequilibrios de conectividad entre las regiones cerebrales, en lugar de aumentar o disminuir la actividad de un área en particular. ${ }^{15}$

En esta misma línea, y bajo la premisa que el hipocampo y la corteza prefrontal (PFC) desempeñan un papel 
Tabla 1: Artículos originales de conectividad cerebral en esquizofrenia.

\begin{tabular}{|c|c|c|c|c|c|c|c|}
\hline Autores & Año & Técnica & Principal hallazgo & Trastorno & Muestra & Medicación & $\begin{array}{l}\text { Gravedad media de } \\
\text { los síntomas }\end{array}$ \\
\hline $\begin{array}{c}\text { Tang } Y, \\
\text { Wang } L, \text { Cao } \\
F \text { \& Tan } L^{16}\end{array}$ & 2012 & $\begin{array}{l}\text { fMRI en } \\
\text { esta- } \\
\text { do de } \\
\text { reposo }\end{array}$ & $\begin{array}{c}\text { Disminución en la } \\
\text { conectividad de: } \\
\text { VN, DMN, SRN y } \\
\text { SMN }\end{array}$ & SZ & $\begin{array}{l}S Z(n=22) \\
C S(n=22)\end{array}$ & $\begin{array}{c}\text { Risperidona ( } n=8, \\
2-4 \text { mg/día), cloza- } \\
\text { pina ( } n=3,200-350 \\
\text { mg/día), quetiapina ( } n \\
=4,400-600 \mathrm{mg} / \mathrm{día}), \\
\text { aripiprazol ( } \mathrm{n}=1,25 \\
\mathrm{mg} / \mathrm{día})\end{array}$ & $\begin{array}{c}\text { PANSS: } \\
75.64 \pm 12.91\end{array}$ \\
\hline $\begin{array}{c}\text { Orban P, } \\
\text { Desseilles } \\
\text { M, Mendrek } \\
\text { A, Bourque } \\
\text { J, Bellec P \& } \\
\text { Stip E }{ }^{17}\end{array}$ & 2017 & $\begin{array}{l}\text { fMRI con } \\
\text { tarea }\end{array}$ & $\begin{array}{l}\text { En pacientes: } \\
\text { disminución de la } \\
\text { conectividad en } \\
\text { corteza prefrontal } \\
\text { medial, cingulado } \\
\text { anterior y posterior, } \\
\text { lóbulo temporal, ló- } \\
\text { bulo IX del cerebelo } \\
\text { y corteza premotora }\end{array}$ & $S Z$ & $\begin{array}{l}S Z(n=30) \\
C S(n=30)\end{array}$ & $\begin{array}{c}\text { Pacientes con al } \\
\text { menos } 1 \text { medicación } \\
\text { antipsicótica atípica } \\
\text { (clozapina, olanzapina, } \\
\text { quetiapina, risperidona } \\
\text { y/o ziprasidona). Dosis } \\
\text { equivalente promedio } \\
\text { de clorpromazina fue } \\
579.92 \pm 349.38\end{array}$ & $\begin{array}{c}\text { PANSS: } \\
79.83 \pm 20.18 \\
\text { PANSS-P: } \\
18.66 \pm 6.71 \\
\text { PANSS-N: } \\
20.41 \pm 6.82 \\
\text { PANSS-EG: } \\
40.76 \pm 9.82\end{array}$ \\
\hline $\begin{array}{l}\text { Skåtun KC, } \\
\text { Kaufmann } \\
\text { T, Doan NT, } \\
\text { Alnæs D, } \\
\text { Córdova- } \\
\text { Palomera A, } \\
\text { Jönsson EG } \\
\text { \& Andreas- } \\
\text { sen } \mathrm{OA}^{18}\end{array}$ & 2017 & $\begin{array}{l}\text { fMRI en } \\
\text { esta- } \\
\text { do de } \\
\text { reposo }\end{array}$ & $\begin{array}{l}\text { Reducciones } \\
\text { robustas en la co- } \\
\text { nectividad funcional } \\
\text { en los trastornos } \\
\text { del espectro SZ, } \\
\text { lo que indica un } \\
\text { flujo de información } \\
\text { interrumpido en las } \\
\text { regiones sensoria- } \\
\text { les, subcorticales y } \\
\text { frontales del cerebro }\end{array}$ & SZ & $\begin{array}{c}\text { Estudio TOP: } \\
\text { SZ }(n=96) \\
\text { CS }(n=277) \\
\text { KaSP: } \\
\text { SZ }(n=52) \\
\text { CS }(n=30) \\
\text { HUBIN: } \\
\text { SZ }(n=34) \\
\text { CS }(n=41)\end{array}$ & $\begin{array}{c}\text { DDD antipsicóticos } \\
\text { Estudio TOP: } 1.31 \\
(1.2) \\
\text { KaSP: } 1.11(0.7) \\
\text { HUBIN: } 1.26(0.8)\end{array}$ & $\begin{array}{c}\text { Estudio TOP: } \\
\text { PANSS: } \\
58.2 \pm 13.6 \\
\text { PANSS-P: } 13.5 \pm 5 \\
\text { PANSS-N: } \\
14.9 \pm 4.9 \\
\text { KaSP: } \\
\text { PANSS: } \\
74.4 \pm 20.7 \\
\text { PANSS-P: } \\
18.8 \pm 6.1 \\
\text { PANSS-N: } \\
17.4 \pm 7.3 \\
\text { HUBIN: } \\
\text { SANS: } 29.3 \pm 14.9 \\
\text { SAPS: } 8.8 \pm 7.5\end{array}$ \\
\hline Chen $\mathrm{B}^{20}$ & 2019 & $\begin{array}{l}\text { fMRI en } \\
\text { esta- } \\
\text { do de } \\
\text { reposo }\end{array}$ & $\begin{array}{l}\text { A nivel de subsis- } \\
\text { temas cerebrales, } \\
\text { los puntajes TE en } \\
\text { DMN son anormales } \\
\text { en esquizofrenia }\end{array}$ & SZ & $\begin{array}{l}S Z(n=53) \\
C S(n=67)\end{array}$ & No reportan & $\begin{array}{c}\text { PANSS-P: } \\
14.94 \pm 4.60 \\
\text { PANSS-N: } \\
14.43 \pm 5.26 \\
\text { PANSS-EG: } \\
30.07 \pm 8.28\end{array}$ \\
\hline
\end{tabular}

$\mathrm{fMRI}=$ imagen por resonancia magnética funcional; $S Z$ = esquizofrenia; $C S=$ controles sanos; VN = red cortical visual; $D M N=$ red neuronal por defecto; $S R N=$ red mental autorreferencial; $\mathrm{SMN}$ = red sensorial-motora; $\mathrm{dFC}=$ conectividad funcional dinámica; $\mathrm{SFC}=$ conectividad funcional estática; $\mathrm{CHR}=$ individuos con alto riesgo clínico; $\mathrm{dACC}=$ cingulado anterior dorsal bilateral; TE = entropía topológica; PANSS = escala de síndrome positivo y negativo; PANSS-P = escala positiva; PANSS-N = escala negativa; PANSS-EG = escala general; KaSP = proyecto de esquizofrenia Karolinska; HUBIN = informática del cerebro humano; DDD = dosis diaria definida; SAPS = escala de evaluación de síntomas positivos; SANS = escala de evaluación de síntomas negativos. 
Tabla 2: Artículos originales de conectividad cerebral en esquizofrenia y otras enfermedades mentales graves.

\begin{tabular}{|c|c|c|c|c|c|c|c|}
\hline Autores & Año & Técnica & Principal hallazgo & Trastorno & Muestra & Medicación & $\begin{array}{l}\text { Gravedad media de } \\
\text { los síntomas }\end{array}$ \\
\hline $\begin{array}{l}\text { Benedetti } \\
\text { F, Bollettini } \\
\text { I, Radaelli } \\
\text { D, Poletti S, } \\
\text { Locatelli C, } \\
\text { Falini A \& } \\
\text { Colombo C22 }\end{array}$ & 2015 & $\begin{array}{l}\text { fMRI en } \\
\text { esta- } \\
\text { do de } \\
\text { reposo }\end{array}$ & $\begin{array}{l}\text { Correlación inversa } \\
\text { entre la gravedad } \\
\text { de las medidas de } \\
\text { ACE y cambios } \\
\text { en las medidas de } \\
\text { DTI de FA, y una } \\
\text { correlación positiva } \\
\text { con MD en varios } \\
\text { tractos de WM }\end{array}$ & $\begin{array}{l}\text { SZ con } \\
\text { antece- } \\
\text { dentes } \\
\text { de } \\
\text { maltrato } \\
\text { infantil }\end{array}$ & $S Z(n=83)$ & $\begin{array}{l}\text { Carga de medica- } \\
\text { ción: } 335.31 \pm 188.98\end{array}$ & $\begin{array}{c}\text { PANSS-P: } \\
18.12 \pm 5.26 \\
\text { PANSS-N: } \\
20.8 \pm 4.69 \\
\text { PANSS-EG: } \\
39.27 \pm 8.32 \\
\text { FRQ: } 26.33 \pm 9.54\end{array}$ \\
\hline $\begin{array}{l}\text { Davey CG, } \\
\text { Whittle S, } \\
\text { Harrison } \\
\text { BJ, Sim- } \\
\text { mons JG, } \\
\text { Byrne ML, } \\
\text { Schwartz OS } \\
\text { \& Allen NB }{ }^{23}\end{array}$ & 2015 & $\begin{array}{l}\text { fMRI en } \\
\text { esta- } \\
\text { do de } \\
\text { reposo }\end{array}$ & $\begin{array}{l}\text { La conectividad } \\
\text { Amígdala-sACC } \\
\text { está asociada con } \\
\text { NA en la adoles- } \\
\text { cencia }\end{array}$ & $\begin{array}{l}\text { NAy } \\
\text { primer } \\
\text { episodio } \\
\text { depre- } \\
\text { sivo }\end{array}$ & $\begin{array}{l}56 \text { ado- } \\
\text { lescentes } \\
\text { sanos }\end{array}$ & No reportan & $\begin{array}{c}\text { Con MDD: } \\
\text { CES-D = 17.7 } \\
\text { (S.D. = 11.7) } \\
\text { Sin MDD: } \\
\text { CES-D = 7.5 } \\
\text { (S.D. }=7.7)\end{array}$ \\
\hline $\begin{array}{l}\text { Kaufmann } \\
\text { T, Alnæs D, } \\
\text { Brandt CL, } \\
\text { Doan NT, } \\
\text { Kauppi K, } \\
\text { Bettella F \& } \\
\text { Melle IS }{ }^{24}\end{array}$ & 2017 & $\begin{array}{l}\text { fMRI con } \\
\text { tarea }\end{array}$ & $\begin{array}{l}\text { Correlaciones } \\
\text { positivas entre } \\
\text { tareas y perfiles } \\
\text { de conectividad de } \\
21 \text { componentes } \\
\text { independientes, } \\
\text { entre ellos: DMN, } \\
\text { tálamo, hipocampo, } \\
\text { amígdala e insula }\end{array}$ & SZ y TAB & $\begin{array}{l}\mathrm{SZ}(n=90) \\
\operatorname{TAB}(n=97) \\
\operatorname{CS}(n=136)\end{array}$ & $\begin{array}{c}\text { SZ: antipsicóticos } \\
(n=61), \text { antide- } \\
\text { presivos }(n=21), \\
\text { antiepilépticos } \\
(n=5) \text {, ansiolíticos } \\
(n=7) \\
\text { TAB: antipsicóticos } \\
(n=27) \text {, antide- } \\
\text { presivos }(n=22), \\
\text { antiepilépticos } \\
(n=47), \text { ansiolíticos } \\
(n=5)\end{array}$ & No reportan \\
\hline $\begin{array}{l}\text { Boissoneault } \\
\text { J, Letzen J, } \\
\text { Lai S, Rob- } \\
\text { inson ME \& } \\
\text { Staud R }{ }^{25}\end{array}$ & 2018 & $\begin{array}{l}\text { fMRI en } \\
\text { esta- } \\
\text { do de } \\
\text { reposo }\end{array}$ & $\begin{array}{c}\text { CS tenía dFC } \\
\text { más alto que SFC } \\
\text { para conectividad } \\
\text { entre hipocampo } \\
\text { y lóbulo parietal } \\
\text { superior derecho; } \\
\text { conectividad en las } \\
\text { estructuras occipi- } \\
\text { tales y temporales } \\
\text { disminuyó en CS } \\
\text { pero aumentó en } \\
\text { SFC }\end{array}$ & SFC & $\begin{array}{l}\operatorname{SFC}(n=19) \\
\operatorname{CS}(n=15)\end{array}$ & No reportan & $\begin{array}{c}\text { Pennebaker Inven- } \\
\text { tory of Limbic Lan- } \\
\text { guidness m (sd) } \\
\text { CS = } 80.33(19.57) \\
\text { SFC }=136.88 \\
(32.68)\end{array}$ \\
\hline
\end{tabular}

fMRI = imagen por resonancia magnética funcional; $\mathrm{SZ}$ = esquizofrenia; $\mathrm{TAB}=$ trastorno afectivo bipolar; $\mathrm{CS}$ = controles sanos; $\mathrm{CHR}=$ individuos con alto riesgo clínico; $\mathrm{SFC}=$ síndrome de fatiga crónica; $\mathrm{VN}=$ red cortical visual; $\mathrm{DMN}=$ red neuronal por defecto; $\mathrm{SRN}=$ red mental autorreferencial; $\mathrm{SMN}=$ red sensorial-motora; $\mathrm{sgACC}$ FC = cingulado subgenual; $\mathrm{ACE}=$ experiencias infantiles adversas; $\mathrm{DTI}=$ imágenes del tensor de difusión; $\mathrm{FA}=$ anisotropía fraccional; $\mathrm{MD}=$ difusividad media; $\mathrm{WM}=$ materia blanca; $\mathrm{Na}=$ afectividad negativa; $T E$ = entropía topológica; $\mathrm{dFC}=$ conectividad funcional dinámica; $\mathrm{SFC}=$ conectividad funcional estática; PANSS = escala de síndrome positivo y negativo; PANSS-P = escala positiva; PANSS-N = escala negativa; PANSS-EG = escala general; $S C R=$ detección de trastornos emocionales relacionados con la ansiedad infantil; $C D I=$ inventario de depresión infantil; RFQ = cuestionario de familias riesgosas; CES-D = escala de depresión de Radloff; MMD = trastorno depresivo mayor; $S C R=$ detección de trastornos emocionales relacionados con la ansiedad infantil; $\mathrm{CDI}=$ inventario de depresión infantil. 
central en diversas funciones cognitivas y de comportamiento, y que las interacciones hipocampales-prefrontales están alteradas en la enfermedad psiquiátrica y pueden contribuir a su fisiopatología, Sigurdsson y Duvarci brindan una visión general del papel de las interacciones hipocampales-prefrontales en la salud y la enfermedad, a partir de una revisión. ${ }^{26}$ En concreto, describen estudios donde se encontraron alteraciones, especialmente en esquizofrenia, que sugieren que el circuito hipocampal-prefrontal juega un papel importante en diversas funciones cognitivas y emocionales, además el PFC, en particular su subregión dorsolateral, subyace a muchas de las funciones cognitivas que están alteradas en la esquizofrenia y, por lo tanto, ha sido un foco importante de investigación en la fisiopatología de la enfermedad.

Avanzando en nuestra consideración, en la revisión que realizan, mencionan que más allá de la actividad anormal de las áreas cerebrales, se cree que las interacciones funcionales aberrantes entre las regiones del cerebro podrían deberse a una conectividad funcional anormal que también depende del contexto, elemento que no había aparecido en ninguno de los artículos revisados y que supone un gran aporte a la comprensión tanto de la psicopatología de la esquizofrenia como a los síntomas cognitivos. Al respecto estos autores puntualizan que la resonancia magnética funcional en estado de reposo caracteriza la conectividad cerebral funcional intrínseca y que estas imágenes han revelado una desconectividad funcional generalizada entre regiones cerebrales, que también se sabe que exhiben niveles anormales de activación en relación con impedimentos en el desempeño de tareas y/o sintomatología, y finalmente proponen que esta cuestión de la independencia contextual de la conectividad funcional anormal en pacientes con esquizofrenia puede abordarse mejor caracterizando tales alteraciones utilizando paradigmas conductuales bien definidos. ${ }^{14}$

\section{DISCUSIÓN}

Los mecanismos biológicos que se han propuesto para entender los trastornos mentales, principalmente del estado de ánimo, por lo general no habían incluido un componente neuropatológico. Sin embargo, una importante parte de la literatura de resonancia magnética ha descrito anormalidades no sólo estructurales en las regiones, sino de las conexiones cerebrales, lo que ha alentado más investigaciones neuropatológicas. En esta línea se plantea que existen dos factores que podrían explicar el interés neuropatológico actual en el trastorno del estado de ánimo y proporcionan el contexto para entender estos estudios; el primero, es que se refleja un creciente énfasis neurobiológico en la explicación de los trastornos psiquiátricos en general y, más específicamente, el progreso reciente en el esclarecimiento de la neuropatología de la esquizofrenia, que ha alentado este enfoque. ${ }^{27}$ En segundo lugar, la evidencia de IRM de anormalidades cerebrales estructurales en trastornos del estado de ánimo que se ha acumulado constantemente en los últimos años, dando un impulso a la búsqueda de sus correlatos histológicos y celulares.

Es por esto que en un momento en el que se encuentra en discusión la clasificación de los trastornos mentales, aparece la aplicación de algunos métodos de conectividad cerebral para la evaluación tanto de la enfermedad mental como del riesgo psiquiátrico en la población. ${ }^{28}$ Muchos de los profesionales del campo de la salud mental creen que la noción de trastornos mentales categóricos y discretos, originalmente articulados por los criterios de diagnóstico de investigación y codificados en los manuales diagnósticos, está tan alejada de la realidad biológica que impide descubrimientos científicos que podrían resultar clínicamente útiles. Estos sistemas de diagnóstico psiquiátrico se justifican principalmente en criterios que se derivan únicamente de la observación del clínico, el autoinforme del paciente y el curso de la enfermedad. ${ }^{10}$

Uno de los principales hallazgos que reporta la bibliografía revisada es que la enfermedad mental está asociada con patrones de conectividad ineficiente, traducida en una conectividad intrínseca amplia entre la corteza de asociación visual y las redes que apoyan el control ejecutivo y los procesos autorreferenciales, redes que a menudo se ven afectadas por trastornos categóricos que incluyen esquizofrenia, depresión y trastorno bipolar. ${ }^{29}$ Por su parte, otros autores han propuesto que la esquizofrenia está asociada con una desconectividad frontotemporal; sin embargo, no está claro si éste es un factor de riesgo para el desarrollo del trastorno o si es una consecuencia de la enfermedad establecida que se desarrolla en el curso de la misma. ${ }^{30}$ No obstante, se ha descrito que el trastorno bipolar y la esquizofrenia comparten mecanismos de riesgo genético, y hay informes de similitudes en las alteraciones de los circuitos cerebrales y la conectividad, que están asociadas con estos dos trastornos. ${ }^{31}$

Es importante resaltar que las imágenes de resonancia magnética funcional (fMRI) nos permiten detectar diferencias en la activación de distintas áreas del cerebro que previamente han sido descritas como alteradas en cada uno de los trastornos mentales graves, puesto que, en los síntomas característicos de cada una de las enfermedades mentales, se ha encontrado, de manera bastante consistente, aumentos o disminución en la activación de diferentes áreas, entendiendo además que clarificar la base neural de los trastornos psiquiátricos comunes, puede guiar el desarrollo de nuevas estrategias preventivas y terapéuticas.

Sin embargo, es necesario tener en cuenta que los individuos tienen patrones únicos de conectividad funcional, por lo tanto, para usar resultados de conectividad como 
biomarcadores útiles, estas diferencias individuales deben ser relevantes y junto con otras variables sociodemográficas, conductuales y clínicas, permitir la comparación con perfiles similares para ayudar a predecir la probabilidad de varios resultados de salud; además, se debe establecer la validez de estos perfiles individuales, así como su relación significativa con el comportamiento. ${ }^{32}$

En definitiva, un paso clave hacia el desarrollo de un sistema de diagnóstico y tratamiento para enfermedades mentales basado en la neuropatofisiología es caracterizar los circuitos cerebrales que subyacen en los dominios críticos de la función social, cognitiva y afectiva que se interrumpen en los trastornos psiquiátricos. Por su parte, Hiser y Koenigs mencionan que uno de los aspectos más desafiantes de la atención al paciente psiquiátrico es la heterogeneidad sustancial presente dentro de un único diagnóstico categórico, un ejemplo claro son los criterios diagnósticos estándar para la depresión mayor, que además se pueden cumplir con muchas más combinaciones de síntomas. ${ }^{33}$ La aclaración de los subtipos dentro de un diagnóstico psiquiátrico es una gran promesa para una atención al paciente más precisa y efectiva, es por esto que se propone que estudios basados en la neurobiología se correspondan más estrechamente con las diferencias en los mecanismos proximales de la disfunción y, por lo tanto, produzcan grupos de diagnóstico con necesidades de tratamiento más homogéneas.

\section{CONCLUSIONES}

Las altas tasas de comorbilidad de la enfermedad mental, el riesgo que comparten entre ellas y los mecanismos terapéuticos superpuestos hacen necesario que la investigación en psicopatología transite hacia investigaciones transdiagnósticas de síntomas agrupados, lo que ayudaría a mejorar la comprensión de los fenómenos asociados con la enfermedad mental. Además, si se consigue caracterizar la conectividad cerebral en los diferentes trastornos, se podrían proponer como biomarcadores, lo que impactaría el proceso de diagnóstico psiquiátrico, mejorándolo y convirtiéndose en un desafío clave en la neurociencia clínica. De hecho, el papel de la disfunción cerebral en la patología psiquiátrica no fue inicialmente evidente debido a las pocas herramientas disponibles para la investigación.

En vista de que los trastornos psiquiátricos como la esquizofrenia, la depresión mayor y el trastorno bipolar siguen siendo un enigma para los científicos desde que se definieron por primera vez hace más de un siglo, y que son considerados como enfermedades médica graves responsables de una considerable morbilidad y discapacidad en la población, se hace necesario comprender los fundamentos neuropatológicos de estos trastornos, suponiendo un paso esencial hacia el desarrollo de tratamientos específicos, teniendo en cuenta, además, que la conectividad funcional en estado de reposo es una herramienta poderosa en neurociencia clínica, porque se puede administrar fácilmente en las poblaciones de pacientes, demuestra una estabilidad similar a un rasgo, así como una heredabilidad moderada, y representa una potente sonda de intrínseca arquitectura de redes neuronales que juegan un papel principal en la configuración de la actividad de red basada en tareas y los comportamientos asociados. ${ }^{29}$

En consecuencia, el estudio de las regiones anormales y los subsistemas que las componen, de las cuales ya se tiene conocimiento que desencadenan trastornos complejos de procesamiento emocional y cognitivo, serán un recurso efectivo para mejorar el diagnóstico de enfermedad mental en el campo de investigación en neurociencias, considerando que los trastornos mentales graves en general presentan patrones característicos de deficiencias emocionales, cognitivas y sociales. ${ }^{34}$

\section{AGRADECIMIENTOS}

Al ministerio de ciencia y tecnología de Colombia por el apoyo a través de la beca Colciencias 757 de 2016 para doctorados nacionales.

\section{REFERENCIAS}

1. Charney D, Buxbaum J, Sklar P, Nestler E. Neurobiology of mental illness. 4th ed. New York: Oxford University Press; 2013.

2. Tzourio-Mazoyer N, Landeau B, Papathanassiou D, Crivello F, Etard $\mathrm{O}$, Delcroix $\mathrm{N}$ et al. Automated anatomical labeling of activations in SPM using a macroscopic anatomical parcellation of the MNI MRI single-subject brain. Neuroimage [Internet]. 2002; 15 (1): 273-289. Available in: http://www.ncbi.nlm.nih.gov/pubmed/11771995

3. Biswal B, Zerrin Y, Haughton VM, Hyde JS. Functional connectivity in the motor cortex of resting human brain using echo-planar MRI. Magn Reson Med. 1995; 34 (4): 537-541.

4. Friston KJ, Frith CD. Schizophrenia: a disconnection syndrome? Vol. 3. New York, N.Y.: Clinical Neuroscience; 1995. pp. 89-97.

5. Robledo Giraldo S, Osorio Zuluaga GA, López Espinosa C. Networking en pequeña empresa: una revisión bibliográfica utilizando la teoría de grafos. Rev Vinculos. 2014; 6 (2): 6-16.

6. Hirsch JE. An index to quantify an individual's scientific research output. Proc Natl Acad Sci U S A. 2005; 102 (46): 16569-16572.

7. Insel T, Cuthbert B, Garvey M et al. Research domain criteria (RDoC): toward a new classification framework for research on mental disorders. Am J Psychiatry. 2010; 167 (7): 748-751.

8. Buckner RL, Andrews-Hanna JR, Schacter DL. The brain's default network: anatomy, function, and relevance to disease. Ann N Y Acad Sci. 2008; 1124: 1-38.

9. Walterfang M, Wood SJ, Velakoulis D, Pantelis C. Neuropathological, neurogenetic and neuroimaging evidence for white matter pathology in schizophrenia. Neurosci Biobehav Rev. 2006; 30 (7): 918-948.

10. Buckholtz JW, Meyer-Lindenberg A. Psychopathology and the human connectome: toward a transdiagnostic model of risk for mental illness. Neuron. 2012; 74 (6): 990-1004. doi: 10.1016/j.neuron.2012.06.002.

11. Anticevic A, Cole MW, Murray JD, Corlett PR, Wang XJ, Krystal JH. The role of default network deactivation in cognition and disease. 
Trends Cogn Sci [Internet]. 2012; 16 (12): 584-592. Available in: http://dx.doi.org/10.1016/j.tics.2012.10.008

12. Anticevic A, Cole MW, Repovs G, Murray JD, Brumbaugh MS, Winkler AM et al. Characterizing thalamo-cortical disturbances in schizophrenia and bipolar illness. Cereb Cortex. 2014; 24 (12): 3116-3130.

13. Lynall ME, Bassett DS, Kerwin R, McKenna PJ, Kitzbichler M, Muller $U$ et al. Functional connectivity and brain networks in schizophrenia. J Neurosci [Internet]. 2010; 30 (28): 9477-9487. Available in: http://www.jneurosci.org/cgi/doi/10.1523/JNEUROSCl.0333-10.2010

14. Baeken C, Marinazzo D, Everaert H, Wu GR, Van Hove C, Audenaert $\mathrm{K}$ et al. The impact of accelerated HF-rTMS on the subgenual anterior cingulate cortex in refractory unipolar major depression: Insights from 18FDG PET brain imaging. Brain Stimul [Internet]. 2015; 8 (4): 808-815. Available in: http://dx.doi.org/10.1016/j. brs.2015.01.415

15. Pandya M, Altinay M, Malone DA, Anand A. Where in the brain is depression? Curr Psychiatry Rep. 2012; 14 (6): 634-642.

16. Tang Y, Wang L, Cao F, Tan L. Identify schizophrenia using restingstate functional connectivity: an exploratory research and analysis. Biomed Eng Online [Internet]. 2012; 11: 50. Available in: http:// www.embase.com/search/results?subaction $=$ viewrecord\&from $=e$ xport\&id = L366368941\%0Ahttp://ad4mh3sr7v.search.serialssolutions.com? sid $=$ EMBASE\&issn $=1475925$ X\&id $=$ doi: \&atitle $=$ Identif $y+$ schizophrenia + using + resting-state + functional + connectivity $\%$ $3 \mathrm{~A}+$ an+explorator

17. Orban P, Desseilles M, Mendrek A, Bourque J, Bellec P, Stip E. Altered brain connectivity in patients with schizophrenia is consistent across cognitive contexts. J Psychiatry Neurosci. 2017; 42 (1): 1726.

18. Skåtun KC, Kaufmann T, Doan NT, Alnæs D, Córdova-Palomera A, Jönsson EG et al. Consistent functional connectivity alterations in schizophrenia spectrum disorder: a multisite study. Schizophr Bull. 2017; 43 (4): 914-924.

19. Fryer SL, Roach BJ, Ford JM, Donaldson KR, Calhoun VD, Pearlson GD et al. Should I stay or should I go? FMRI study of response inhibition in early illness schizophrenia and risk for psychosis. Schizophr Bull. 2019; 45 (1): 158-168.

20. Chen B. Abnormal cortical region and subsystem complexity in dynamical functional connectivity of chronic schizophrenia: a new graph index for fMRI analysis. J Neurosci Methods [Internet]. 2019; 311: 28-37. Available in: https://doi.org/10.1016/j.jneumeth.2018.10.011

21. Thomason ME, Marusak HA, Tocco MA, Vila AM, McGarragle O, Rosenberg DR. Altered amygdala connectivity in urban youth exposed to trauma. Soc Cogn Affect Neurosci. 2015; 10 (11): 14601468.

22. Benedetti F, Bollettini I, Radaelli D, Poletti S, Locatelli C, Falini A et al. Adverse childhood experiences influence white matter micros- tructure in patients with bipolar disorder. Psychol Med. 2015; 44 (14): 3069-3082.

23. Davey CG, Whittle S, Harrison BJ, Simmons JG, Byrne ML, Schwartz OS et al. Functional brain-imaging correlates of negative affectivity and the onset of first-episode depression. Psychol Med. 2015; 45 (5): 1001-1009.

24. Kaufmann T, Alnæs D, Brandt CL, Doan NT, Kauppi K, Bettella $\mathrm{F}$ et al. Task modulations and clinical manifestations in the brain functional connectome in $1615 \mathrm{fMRI}$ datasets. Neuroimage [Internet]. 2017; 147: 243-252. Available in: http://dx.doi.org/10.1016/j. neuroimage.2016.11.073

25. Boissoneault J, Letzen J, Lai S, Robinson ME, Staud R. Static and dynamic functional connectivity in patients with chronic fatigue syndrome: use of arterial spin labelling fMRI. Clin Physiol Funct Imaging. 2018; 38 (1): 128-137.

26. Sigurdsson T, Duvarci S. Hippocampal-prefrontal interactions in cognition, behavior and psychiatric disease. Front Syst Neurosci. 2016; 9: 190.

27. Harrison PJ. The neuropathology of primary mood disorder. Brain. 2002; 125 (7): 1428-1449.

28. Hyman SE. The diagnosis of mental disorders: the problem of reification. T Annu Rev Clin Psychol. 2010; 6: 155-179.

29. Elliott ML, Romer A, Knodt AR, Hariri AR. A connectome-wide functional signature of transdiagnostic risk for mental illness. Biol Psychiatry [Internet]. 2018; 84 (6): 452-459. Available in: https:// doi.org/10.1016/j.biopsych.2018.03.012

30. Allen P, Stephan KE, Mechelli A, Day F, Ward N, Dalton J et al. Cingulate activity and fronto-temporal connectivity in people with prodromal signs of psychosis. Neuroimage [Internet]. 2010; 49 (1): 947-955. Available in: http://dx.doi.org/10.1016/j.neuroimage.2009.08.038

31. Anticevic A, Brumbaugh MS, Winkler AM, Lombardo LE, Barrett J, Corlett PR et al. Global prefrontal and fronto-amygdala dysconnectivity in bipolar I disorder with psychosis history. Biol Psychiatry. 2012; 73 (6): 565-573.

32. Finn ES, Todd Constable R. Individual variation in functional brain connectivity: implications for personalized approaches to psychiatric disease. Dialogues Clin Neurosci. 2016; 18 (3): 277-287.

33. Hiser J, Koenigs $M$. The multifaceted role of the ventromedial prefrontal cortex in emotion, decision making, social cognition, and psychopathology. Biol Psychiatry [Internet]. 2018; 83 (8): 6386347. Available in: https://doi.org/10.1016/j.biopsych.2017.10.030

34. Pasternak O, Kelly S, Sydnor VJ, Shenton ME. Advances in microstructural diffusion neuroimaging for psychiatric disorders. Neuroimage [Internet]. 2018; 182: 259-282. Available in: https://doi. org/10.1016/j.neuroimage.2018.04.051

Conflicto de intereses: Las autoras declaran no tener conflicto de intereses. 